\title{
Singularity analysis for integrable systems by their mirrors
}

\author{
Jishan $\mathrm{Hu}$ and Min Yan \\ Department of Mathematics, The Hong Kong University of Science and Technology, Clear Water \\ Bay, Kowloon, Hong Kong \\ E-mail: majhu@uxmail.ust.hk and mamyan@uxmail.ust.hk
}

Received 13 November 1998, in final form 8 June 1999

Recommended by V F Lazutkin

\begin{abstract}
We use the Lorenz system, the Rikitake model and the nonlinear Schrödinger equation to demonstrate that for completely integrable systems, there exist what we call regular mirror systems near movable singularities. The method for finding the mirror systems is very similar to the original Weiss et al's (1983 J. Math. Phys. 24 522-6) version of the Painleve test. It tests the complete integrability and gives a systematic and conceptual proof that the formal Laurent series generated by the Painlevé test are convergent.
\end{abstract}

AMS classification scheme numbers: 35Q51, 58F07

\section{Introduction}

When Painlevé [5] studied movable singularities of solutions of the equation

$$
u^{\prime \prime}=6 u^{2}+x,
$$

he introduced two new dependent variables $\theta$ and $\xi$ by

$$
u=\theta^{-2}, \quad \theta^{\prime}=1+\frac{1}{4} x \theta^{4}+\frac{1}{4} \theta^{5}-\frac{1}{2} \theta^{6} \xi,
$$

and converted the equation into the following regular system:

$$
\begin{aligned}
& \frac{\mathrm{d} \theta}{\mathrm{d} x}=1+\frac{1}{4} x \theta^{4}+\frac{1}{4} \theta^{5}-\frac{1}{2} \theta^{6} \xi \\
& \frac{\mathrm{d} \xi}{\mathrm{d} x}=\frac{1}{8} x^{2} \theta+\frac{3}{8} x \theta^{2}+\theta^{3}\left(\frac{1}{4}-x \xi\right)-\frac{5}{4} \theta^{4} \xi-\frac{3}{2} \theta^{5} \xi^{2} .
\end{aligned}
$$

This differential system (which we called the mirror system in [3,4]) determines the behaviours of solutions near their algebraic movable singularities. The regularity of the system makes it possible to apply the Cauchy-Kowalevski theorem. This implies that the solutions of (1.1) must be meromorphic near algebraic movable singularities.

Our recent work [3] has demonstrated that, for single high-order equations, what Painlevé did a century ago is a general property. In fact, we will argue in [4] that the existence of regular mirror systems is equivalent to the Painlevé test $[1,8]$, which has been the most effective algorithm for detecting complete integrability. Moreover, we can use the mirror system to show that the formal Laurent series solutions obtained from the Painlevé test must be convergent, and thereby justify the Painlevé test.

The first purpose of this paper is to demonstrate how to apply a similar idea to systems of differential equations. Secondly, we have gained much insight in our algorithm since we 
finished [3], and we would like to take the opportunity to present our algorithm in a more understandable way (and to incorporate the necessary changes needed for systems). We will work out the details for the examples of the Lorenz system, the Rikitake model and the nonlinear Schrödinger (NLS) equation. The treatment of the Lorenz system is the most standard. The Rikitake model has a double root as resonance. The NLS equation is a partial differential equation (PDE), with one resonance parameter appearing as leading behaviour.

Our construction of the mirror system is conducted under the best scenario assumption. As we will argue in [4], successfully carrying out our construction is in fact equivalent to passing the Painlevé test (meaning that there is a high enough number of non-negative resonances). It is quite possible that we can still construct some sort of partial mirror system in cases such as insufficient number of resonances, negative resonances, or fractional resonances. We will try to explore these in future research.

\section{Algorithm for mirror systems}

Our algorithm for finding the mirror systems for differential systems is based on a computation similar to the original version of Weiss et al's Painlevé test for PDEs [8]. We outline the steps as follows, using a first-order ordinary differential equation (ODE) system in dependent variables $X, Y$ and $Z$ as an example.

Step 1. Find the dominant behaviour in the neighbourhood of the movable singularity.

Step 2. Introduce indicial normalization by writing one dependent variable in the form $\theta^{-k}$, where $k$ is the leading exponent of the dependent variable.

Suppose our ODE system has the following dominant behaviour:

$$
X=X_{0}\left(t-t_{0}\right)^{-k}, \quad Y=Y_{0}\left(t-t_{0}\right)^{-l}, \quad Z=Z_{0}\left(t-t_{0}\right)^{-m}
$$

near movable singularities (only one of $k, l$ and $m$ needs to be positive). Then we may choose a positive leading exponent, say $k$, and introduce indicial normalization $X=\theta^{-k}$.

Step 3. Find formal Laurent series of $\theta^{\prime}$ and the other dependent variables in powers of $\theta$.

For our ODE system, we are looking for the following formal Laurent $\theta$-series:

$$
\begin{aligned}
& \theta^{\prime}=a_{0}+a_{1} \theta+a_{2} \theta^{2}+\cdots, \\
& Y=\theta^{-l}\left(b_{0}+b_{1} \theta+b_{2} \theta^{2}+\cdots\right), \\
& Z=\theta^{-m}\left(c_{0}+c_{1} \theta+c_{2} \theta^{2}+\cdots\right),
\end{aligned}
$$

where $a_{*}, b_{*}$ and $c_{*}$ are functions of $t$ (but not $t_{0}$ ). The way to find these is similar to the Painlevé test. The leading coefficients $a_{0}, b_{0}$ and $c_{0}$ can be found from the dominant balance equations. The latter coefficients are computed from a linear recursive relation obtained by substituting the series (2.1) into the system.

There is one important difference between our algorithm and the Painlevé test: the function $\phi$ used in [8] to define the singularity manifold depends only on the singularity, and is independent of the resonance parameters. In our algorithm, the function $\theta$ is 'equivalent' to the solution, and has the resonance parameters implicitly built in. This is reflected in that, in substituting (2.1) into the original system, we need to use

$$
X^{\prime}=-k \theta^{-k-1} \theta^{\prime}=-k \theta^{-k-1}\left(a_{0}+a_{1} \theta+a_{2} \theta^{2}+\cdots\right),
$$

and

$$
\begin{gathered}
Y^{\prime}=\theta^{-l}\left(b_{0}^{\prime}+b_{1}^{\prime} \theta+b_{2}^{\prime} \theta^{2}+\cdots\right)+\theta^{-l-1}\left((-l) b_{0}+(-l+1) b_{1} \theta\right. \\
\left.+(-l+2) b_{2} \theta^{2}+\cdots\right)\left(a_{0}+a_{1} \theta+a_{2} \theta+\cdots\right)
\end{gathered}
$$

and similarly for $Z^{\prime}$. 
For autonomous systems, the coefficients $a_{*}, b_{*}$ and $c_{*}$ are constants. This makes the computation a little easier.

For our ODE system, the determinant of the coefficient matrix in the recursive relation is a polynomial of degree three. It has the same roots (resonances) as the determinant in the Painlevé test. The compatibility in our sense is equivalent to the compatibility in the Painlevé test. If $j$ is the largest resonance, then we may stop the computation until $a_{j}, b_{j}$ and $c_{j}$ are found.

Step 4. Truncate the Laurent $\theta$-series of the dependent variables at the resonances one after another by introducing new variables.

For the third-order system, we expect to get two resonance parameters $r_{1}$ and $r_{2}$ at resonances $j_{1} \leqslant j_{2}$. Suppose we have

$$
Y=\theta^{-l}\left(b_{0}+b_{1} \theta+b_{2} \theta^{2}+\cdots+\left(p_{1} r_{1}+q_{1}\right) \theta^{j_{1}}+\cdots\right),
$$

with $p_{1} \neq 0$ (if this does not happen for $Y$, then this happens for $Z$ ). Then we introduce $\xi$ by truncating $Y$ at $r_{1}$

$$
Y=\theta^{-l}\left(b_{0}+b_{1} \theta+b_{2} \theta^{2}+\cdots+\xi \theta^{j_{1}}\right) .
$$

Moreover, we may convert

$$
\xi=p_{1} r_{1}+q_{1}+b_{j_{1}+1} \theta+b_{j_{1}+2} \theta^{2}+\cdots,
$$

and get

$$
r_{1}=p_{1}^{-1}\left(\xi-q_{1}\right)+\beta_{1} \theta+\beta_{2} \theta^{2}+\cdots .
$$

Substituting this into the $\theta$-series of $Z$, we get

$$
Z=\theta^{-m}\left(c_{0}+c_{1} \theta+c_{2} \theta^{2}+\cdots+\left(p_{2} r_{2}+q_{2}\right) \theta^{j_{2}}+\cdots\right),
$$

where $p_{2}$ is necessarily nonzero and the coefficients only involve $t, \xi$ and $r_{2}$ (not $t_{0}$ and $r_{1}$ ). Then we introduce $\eta$ by truncating $Z$ at $r_{2}$

$$
Z=\theta^{-m}\left(c_{0}+c_{1} \theta+c_{2} \theta^{2}+\cdots+\eta \theta^{j_{2}}\right) .
$$

Step 5. Convert the original differential system into a differential system (the mirror system) about new variables.

The formulae $X=\theta^{-k},(2.2)$ and (2.3) is a transformation between $(X, Y, Z)$ and $(\theta, \xi, \eta)$. If the system for $(X, Y, Z)$ passes the Painlevé test, then the mirror system for $(\theta, \xi, \eta)$ should be regular [4].

This completes the description of the algorithm.

The method also applies to the higher-order case. We only need to use

$$
\begin{gathered}
X^{\prime \prime}=(-k)(-k-1) \theta^{-k-2} \theta^{2}-k \theta^{k-1} \theta^{\prime \prime}=(-k)(-k-1) \theta^{-k-2}\left(a_{0}+a_{1} \theta+a_{2} \theta^{2}+\cdots\right)^{2} \\
-k \theta^{k-1}\left(a_{0}^{\prime}+a_{1}^{\prime} \theta+a_{2}^{\prime} \theta^{2}+\cdots\right) \\
-k \theta^{k-1}\left(a_{1}+2 a_{2} \theta+\cdots\right)\left(a_{0}+a_{1} \theta+a_{2} \theta^{2}+\cdots\right),
\end{gathered}
$$

and similar formulae for $Y^{\prime \prime}, Z^{\prime \prime}$ and higher derivatives.

The method also applies to PDE systems. The only difference is that the coefficients in the $\theta$-series involve more variables, and we need to use formulae such as

$$
\begin{aligned}
\partial_{y} Y=\theta^{-l}\left(\partial_{y} b_{0}\right. & \left.+\left(\partial_{y} b_{1}\right) \theta+\left(\partial_{y} b_{2}\right) \theta^{2}+\cdots\right) \\
& +\theta^{-l-1}\left((-l) b_{0}+(-l+1) b_{1} \theta+(-l+2) b_{2} \theta^{2}+\cdots\right)\left(\partial_{y} \theta\right) .
\end{aligned}
$$

In particular, the coefficients of the $\theta$-series may involve $\partial_{y} \theta, \partial_{y^{2}} \theta, \ldots$ 


\section{Examples of mirror systems}

The Painlevé test has been used to uncover integrable cases of many systems of physical interest. For example, Segur found four integrable cases for the Lorenz system [7]. More examples can be found in $[2,6]$. In this section, we demonstrate our algorithm by working out some classical examples.

\subsection{The Lorenz system}

The Lorenz system is an autonomous differential system:

$$
\begin{aligned}
& X^{\prime}=\sigma(Y-X), \\
& Y^{\prime}=-X Z+R X-Y, \\
& Z^{\prime}=X Y-B Z,
\end{aligned}
$$

where $\sigma, R$ and $B$ are constants. In general, this system is not integrable and is well known for the chaotic behaviour of its solutions. The Painlevé test gives us four completely integrable cases for the Lorenz system:

(1) $\sigma=0$;

(2) $\sigma=\frac{1}{2}, B=1, R=0$;

(3) $\sigma=1, B=2, R=\frac{1}{9}$;

(4) $\sigma=\frac{1}{3}, B=0, R$ arbitrary.

If $\sigma=0$, then $X$ is a constant and the system is linear. Therefore, we will not investigate the case anymore. The three other cases were found by the Painlevé test. In what follows, we try to carry out our algorithm for the Lorenz system. We will see that we are successful in exactly these three cases.

By dominant balance we find the leading behaviour

$$
X=X_{0}\left(t-t_{0}\right)^{-1}, \quad Y=Y_{0}\left(t-t_{0}\right)^{-2}, \quad Z=Z_{0}\left(t-t_{0}\right)^{-2},
$$

near a movable singularity $t=t_{0}$. This would suggest to introduce the indicial normalization $X=\theta^{-1}$ and try to compute the formal $\theta$-series (2.1), with $l=m=2$ and $a_{*}, b_{*}, c_{*}$ constants (because the system is autonomous).

Substituting (2.1) into the Lorenz system, we get the following equations for the leading coefficients:

$$
a_{0}=-\sigma b_{0}, \quad-2 a_{0} b_{0}=-c_{0}, \quad-2 a_{0} c_{0}=b_{0} ;
$$

and the recursive relation for the higher-order coefficients

$$
\begin{aligned}
& a_{n}+\sigma b_{n}=\delta_{1, n} \sigma, \\
& -2 b_{0} a_{n}+(n-2) a_{0} b_{n}+c_{n}=\delta_{2, n} R-b_{n-1}-\sum_{j=1}^{n-1}(j-2) a_{n-j} b_{j}, \\
& -2 c_{0} a_{n}-b_{n}+(n-2) a_{0} c_{n}=-B c_{n-1}-\sum_{j=1}^{n-1}(j-2) a_{n-j} c_{j} .
\end{aligned}
$$

By solving (3.2), we find two possible branches of leading behaviours

$$
a_{0}= \pm \frac{\mathrm{i}}{2}, \quad b_{0}=\mp \frac{\mathrm{i}}{2 \sigma}, \quad c_{0}=\frac{1}{2 \sigma} .
$$

Substituting these into the coefficient matrix on the left of (3.3), we see that the determinant of the coefficient matrix is $-\frac{1}{4}(n-2)(n-4)$. Therefore, there are two resonances $j=2,4$, and 
we need to find the compatibility condition for each resonance. We will carry this out only for the branch $a_{0}=\mathrm{i} / 2$. The discussion for the other branch is similar.

From the recursive relation (3.3), we find

$$
a_{1}=\frac{1}{3}-\sigma+\frac{2}{3} B, \quad b_{1}=2-\frac{1}{3 \sigma}-\frac{2}{3 \sigma} B, \quad c_{1}=2 \mathrm{i}-\frac{\mathrm{i}}{\sigma} B .
$$

Then for $n=2$, the recursive relation becomes

$$
\begin{aligned}
& a_{2}+\sigma b_{2}=0, \\
& \frac{\mathrm{i}}{\sigma} a_{2}+c_{2}=-1-2 \sigma+\frac{2}{9 \sigma}+2 B+\frac{2}{9 \sigma} B-\frac{4}{9 \sigma} B^{2}+R, \\
& -\frac{1}{\sigma} a_{2}-b_{2}=\frac{2 \mathrm{i}}{3}-2 \mathrm{i} \sigma+\frac{\mathrm{i}}{3} B-\frac{\mathrm{i}}{3 \sigma} B+\frac{\mathrm{i}}{3 \sigma} B^{2} .
\end{aligned}
$$

The system is consistent if and only if

$$
B^{2}+B(-1+\sigma)+2(1-3 \sigma) \sigma=0 .
$$

This means that we need to consider two possibilities

$$
B=2 \sigma, \quad \text { or } \quad B=1-3 \sigma .
$$

Case 1: $B=2 \sigma . \quad$ In this case, we solve (3.4) and get

$$
a_{2}=-\sigma r_{1}, \quad b_{2}=r_{1}, \quad c_{2}=\mathrm{i} r_{1}-\frac{5}{9}+\frac{2}{9 \sigma}+\frac{2 \sigma}{9}+R,
$$

where $r_{1}$ is the first resonance parameter. Then from the recursive relation, we further have

$$
\begin{aligned}
& a_{3}=-\frac{4 \mathrm{i}}{3} \sigma r_{1}-\frac{20 \mathrm{i}}{3} \sigma^{2} r_{1}-\frac{16}{9} \sigma+\frac{40}{9} \sigma^{2}-\frac{16}{9} \sigma^{3}-8 R \sigma^{2}, \\
& b_{3}=-\frac{4 \mathrm{i}}{3} r_{1}+\frac{20 \mathrm{i}}{3} \sigma r_{1}+\frac{16}{9}-\frac{40}{9} \sigma+\frac{16}{9} \sigma^{2}+8 R \sigma, \\
& c_{3}=-4 \sigma r_{1}+\frac{8 \mathrm{i}}{9}-\frac{20 \mathrm{i}}{9} \sigma+\frac{8 \mathrm{i}}{9} \sigma^{2}+4 \mathrm{i} R \sigma .
\end{aligned}
$$

Substituting the known coefficients into the recursive relation for $n=4$, we have

$$
\begin{aligned}
& -a_{4}-\sigma b_{4}=0, \\
& \frac{\mathrm{i}}{\sigma} a_{4}+\mathrm{i} b_{4}+c_{4}=-\frac{16}{9}+\frac{4 \mathrm{i}}{3} r_{1}-\frac{16 \mathrm{i}}{3} \sigma r_{1}-\frac{20 \mathrm{i}}{3} \sigma^{2} r_{1} \\
& +\frac{8}{3} \sigma+\frac{8}{3} \sigma^{2}-\frac{16}{9} \sigma^{3}-8 R \sigma-8 R \sigma^{2}, \\
& -\frac{1}{\sigma} a_{4}-b_{4}+\mathrm{i} c_{4}=-\frac{8 \mathrm{i}}{27}+\frac{4}{3} \sigma r_{1}+\frac{28}{3} \sigma^{2} r_{1} \\
& -\frac{4 \mathrm{i}}{3} \sigma+\frac{44 \mathrm{i}}{9} \sigma^{2}-\frac{56 \mathrm{i}}{27} \sigma^{3}-\frac{4 \mathrm{i}}{3} R \sigma-\frac{28 \mathrm{i}}{3} R \sigma^{2} .
\end{aligned}
$$

The system is consistent for an arbitrary choice of $r_{1}$ if and only if

$$
\begin{aligned}
& 1-11 \sigma+(24-45 R) \sigma^{2}+(-14+9 R) \sigma^{3}+4 \sigma^{4}=0, \\
& 1-3 \sigma+2 \sigma^{2}=0 .
\end{aligned}
$$

Solving these, we have

$$
\sigma=\frac{1}{2}, \quad R=0 ; \quad \text { or } \quad \sigma=1, \quad R=\frac{1}{9} .
$$


Case 1A: $\sigma=\frac{1}{2}, B=1, R=0$. In this case, we solve (3.5) and get

$$
\begin{aligned}
\theta^{\prime} & =\frac{\mathrm{i}}{2}+\frac{1}{2} \theta+\frac{1}{2} r_{1} \theta^{2}-\mathrm{i} r_{1} \theta^{3}-\frac{1}{2} r_{2} \theta^{4}+\cdots, \\
Y & =-\mathrm{i} \theta^{-2}+r_{1}+2 \mathrm{i} r_{1} \theta+r_{2} \theta^{2}+\cdots, \\
Z & =\theta^{-2}+\mathrm{i} r_{1}-2 r_{1} \theta-3 \mathrm{i} r_{1} \theta^{2}+\cdots,
\end{aligned}
$$

where $r_{2}$ is the second resonance parameter.

Now we introduce new variables $\xi$ and $\eta$ from the Laurent $\theta$-series of $Y$ and $Z$. By cutting the $\theta$-series of $Y$ at $r_{1}$, we introduce $\xi$

$$
Y=-\mathrm{i} \theta^{-2}+\xi
$$

From the $\theta$-series of $\xi$, we have

$$
r_{1}=\xi-2 \mathrm{i} \xi \theta-\left(r_{2}+4 \xi\right) \theta^{2}+\cdots .
$$

Substituting this into the $\theta$-series of $Z$, we have

$$
Z=\theta^{-2}+\mathrm{i} \xi-\left(\mathrm{i} r_{2}+3 \mathrm{i} \xi\right) \theta^{2}+\cdots .
$$

By cutting the $\theta$-series of $Z$ at $r_{2}$, we introduce $\eta$

$$
Z=\theta^{-2}+\mathrm{i} \xi+\eta \theta^{2} .
$$

Combining (3.6) and (3.7) with $X=\theta^{-1}$, we have a change of variable $(X, Y, Z) \leftrightarrow(\theta, \xi, \eta)$. Then it is easy to convert the Lorenz system (3.1) into the following:

$$
\begin{aligned}
\theta^{\prime} & =\frac{\mathrm{i}}{2}+\frac{1}{2} \theta-\frac{\xi}{2} \theta^{2} \\
\xi^{\prime} & =-\xi-\eta \theta \\
\eta^{\prime} & =-2 \eta+\xi \eta \theta .
\end{aligned}
$$

This is a mirror system of the Lorenz system in case $1 \mathrm{~A}$ of the branch $\theta^{\prime} \sim \mathrm{i} / 2$. The most important feature for the singularity analysis is that the system is regular near $\theta=0$, which corresponds to the movable singularity of the Lorenz system.

Case 1B: $\sigma=1, B=2, R=\frac{1}{9}$. In this case, we get the following Laurent $\theta$-series:

$$
\begin{aligned}
\theta^{\prime} & =\frac{\mathrm{i}}{2}+\frac{2}{3} \theta-r_{1} \theta^{2}-\frac{16 \mathrm{i}}{3} r_{1} \theta^{3}-r_{2} \theta^{4}+\cdots, \\
Y & =-\frac{\mathrm{i}}{2} \theta^{-2}+\frac{1}{3} \theta^{-1}+r_{1}+\frac{16 \mathrm{i}}{3} r_{1} \theta+r_{2} \theta^{2}+\cdots, \\
Z & =\frac{1}{2} \theta^{-2}+\mathrm{i} r_{1}-4 r_{1} \theta-\frac{32 \mathrm{i}}{3} r_{1} \theta^{2}+\cdots,
\end{aligned}
$$

where $r_{1}$ and $r_{2}$ can be arbitrary. By first cutting $Y$ at $r_{1}$ and then $Z$ at $r_{2}$, we introduce new variables $\xi$ and $\eta$ by

$$
\begin{aligned}
& X=\theta^{-1}, \\
& Y=-\frac{1}{2} \theta^{-2}+\frac{1}{3} \theta^{-1}+\xi, \\
& Z=\frac{1}{2} \theta^{-2}+\mathrm{i} \xi+\frac{4}{3} \xi \theta+\eta \theta^{2} .
\end{aligned}
$$

The transformation converts the Lorenz system (3.1) into the following mirror system:

$$
\begin{aligned}
& \theta^{\prime}=\frac{\mathrm{i}}{2}+\frac{2}{3} \theta-\xi \theta^{2}, \\
& \xi^{\prime}=-\frac{8}{3} \xi-\eta \theta, \\
& \eta^{\prime}=\frac{4}{3} \xi^{3}-2 \eta+2 \xi \eta \theta .
\end{aligned}
$$


Case 2: $B=1-3 \sigma$. The compatibility condition at the resonance $j=4$ leads to the following condition:

$$
\sigma=\frac{1}{3}, \quad B=0, \quad R \text { arbitrary. }
$$

Under this condition, we get the following $\theta$-series:

$$
\begin{aligned}
\theta^{\prime} & =\frac{\mathrm{i}}{2}-\frac{1}{3} r_{1} \theta^{2}-\frac{1}{3} r_{2} \theta^{4}+\cdots, \\
Y & =-\frac{3 \mathrm{i}}{2} \theta^{-2}+\theta^{-1}+r_{1}+r_{2} \theta^{2}+\cdots, \\
Z & =\frac{3}{2} \theta^{-2}+2 \mathrm{i} \theta^{-1}+\mathrm{i} r_{1}-1+R-\frac{4}{3} r_{1} \theta+\cdots
\end{aligned}
$$

Based on these we introduce new variables $\xi$ and $\eta$

$$
\begin{aligned}
& X=\theta^{-1}, \\
& Y=-\frac{3 \mathrm{i}}{2} \theta^{-2}+\theta^{-1}+\xi, \\
& Z=\frac{3}{2} \theta^{-2}+2 \mathrm{i} \theta^{-1}+\mathrm{i} \xi-1+R-\frac{4}{3} \xi \theta+\eta \theta^{2},
\end{aligned}
$$

by cutting the $\theta$-series of $Y$ at $r_{1}$ and then the $\theta$-series of $Z$ at $r_{2}$. Under the transformation, the Lorenz system becomes

$$
\begin{aligned}
\theta^{\prime} & =\frac{i}{2}-\frac{1}{3} \xi \theta^{2}, \\
\xi^{\prime} & =-\eta \theta, \\
\eta^{\prime} & =-\frac{4}{9} \xi^{2}-\frac{4}{3} \eta+\frac{2}{3} \xi \eta \theta .
\end{aligned}
$$

\subsection{The Rikitake model}

The Rikitake model

$$
\begin{aligned}
& X^{\prime}=-\gamma X+\beta Y+Y Z, \\
& Y^{\prime}=-\gamma Y-\beta X+X Z, \\
& Z^{\prime}=-X Y+\alpha,
\end{aligned}
$$

describes Earth's magneto-hydrodynamic dynamo. The dominant balance argument suggests to us the need to introduce the indicial normalization $X=\theta^{-1}$ and to try to find the Laurent $\theta$-series (2.1), with $l=m=1$ and $a_{*}, b_{*}, c_{*}$ constants.

We substitute the $\theta$-series into the system. By comparing the coefficients of powers of $\theta$ on both sides, we get the equations for the leading coefficients

$$
a_{0}=-b_{0} c_{0}, \quad-a_{0} b_{0}=c_{0}, \quad-a_{0} c_{0}=-b_{0},
$$

which gives four possible branches of leading behaviours:

$$
\left(a_{0}, b_{0}, c_{0}\right)=\left\{\begin{array}{l}
(-\mathrm{i}, 1, \mathrm{i}) \\
(-\mathrm{i},-1,-\mathrm{i}) \\
(\mathrm{i}, 1,-\mathrm{i}) \\
(\mathrm{i},-1, \mathrm{i})
\end{array}\right.
$$


We also have the recursive relation

$$
\begin{aligned}
& a_{n}+c_{0} b_{n}+b_{0} c_{n}=\delta_{1, n} \gamma-\beta b_{n-1}-\sum_{j=1}^{n-1} b_{j} c_{n-j}, \\
& -b_{0} a_{n}+(n-1) a_{0} b_{n}-c_{n}=-\delta_{1, n} \beta-\gamma b_{n-1}-\sum_{j=1}^{n-1}(j-1) a_{n-j} b_{j}, \\
& -c_{0} a_{n}+b_{n}+(n-1) a_{0} c_{n}=\delta_{2, n} \alpha-\sum_{j=1}^{n-1}(j-1) a_{n-j} c_{j} .
\end{aligned}
$$

Substituting (3.10) into the coefficient matrix on the left of (3.11), we see that the determinant of the coefficient matrix is $-(n-2)^{2}$. Therefore, there is one double resonance $j=2$. By checking out the compatibility conditions, we find exactly two cases.

From now on, we proceed with the first branch $\left(a_{0}, b_{0}, c_{0}\right)=(-\mathrm{i}, 1, \mathrm{i})$. The discussion for the other branches is similar.

Case 1: $\alpha=0, \beta=0$. We get the following $\theta$-series:

$$
\begin{aligned}
\theta^{\prime} & =-\mathrm{i}-\left(\mathrm{i} r_{1}+r_{2}\right) \theta^{2}+\cdots, \\
Y & =\theta^{-1}+r_{1} \theta+\cdots, \\
Z & =\mathrm{i} \theta^{-1}+\gamma+r_{2} \theta+\cdots .
\end{aligned}
$$

By cutting the $\theta$-series of $Y$ at $r_{1}$ and the $\theta$-series of $Z$ at $r_{2}$ (at the same time), we introduce new variables $\xi$ and $\eta$ :

$$
\begin{aligned}
& X=\theta^{-1}, \\
& Y=\theta^{-1}+\xi \theta, \\
& Z=\mathrm{i} \theta^{-1}+\gamma+\eta \theta .
\end{aligned}
$$

The transformation converts the Rikitake system (3.9) into the following mirror system:

$$
\begin{aligned}
& \theta^{\prime}=-\mathrm{i}-(\mathrm{i} \xi+\eta) \theta^{2}-\gamma \xi \theta^{3}-\xi \eta \theta^{4}, \\
& \xi^{\prime}=-2 \gamma \xi+\mathrm{i} \xi^{2} \theta+\gamma \xi^{2} \theta^{2}+\xi^{2} \eta \theta^{3}, \\
& \eta^{\prime}=-\mathrm{i} \gamma \xi+\eta^{2} \theta+\gamma \xi \eta \theta^{2}+\xi \eta^{2} \theta^{3} .
\end{aligned}
$$

Case 2: $\alpha=0, \gamma=0$. We get the following $\theta$-series:

$$
\begin{aligned}
& \theta^{\prime}=-\mathrm{i}+2 \beta \theta-\left(\mathrm{i} r_{1}+r_{2}\right) \theta^{2}+\cdots, \\
& Y=\theta^{-1}+2 \mathrm{i} \beta+r_{1} \theta+\cdots, \\
& Z=\mathrm{i} \theta^{-1}-\beta+r_{2} \theta+\cdots .
\end{aligned}
$$

By cutting the $\theta$-series of $Y$ at $r_{1}$ and the $\theta$-series of $Z$ at $r_{2}$, we introduce new variables $\xi$ and $\eta$

$$
\begin{aligned}
& X=\theta^{-1}, \\
& Y=\theta^{-1}+2 \mathrm{i} \beta+\xi \theta, \\
& Z=\mathrm{i} \theta^{-1}-\beta+\eta \theta .
\end{aligned}
$$

The corresponding mirror system is

$$
\begin{aligned}
& \theta^{\prime}=-\mathrm{i}+2 \beta \theta-(\mathrm{i} \xi+\eta) \theta^{2}-2 \mathrm{i} \beta \eta \theta^{3}-\xi \eta \theta^{4}, \\
& \xi^{\prime}=-2 \beta \xi-2 \mathrm{i} \beta \eta+\mathrm{i} \xi^{2} \theta+2 \mathrm{i} \beta \xi \eta \theta^{2}+\xi^{2} \eta \theta^{3}, \\
& \eta^{\prime}=\eta^{2} \theta+2 \mathrm{i} \beta \eta^{2} \theta^{2}+\xi \eta^{2} \theta^{3} .
\end{aligned}
$$




\subsection{The NLS equation}

It is well known that the NLS equation

$$
\mathrm{i} u_{t}+u_{x x}-2|u|^{2} u=0
$$

is completely integrable. To find its mirror system, we complexify all variables and write the NLS equation as a system:

$$
\begin{aligned}
& \mathrm{i} u_{t}+u_{x x}-2 u^{2} v=0, \\
& -\mathrm{i} v_{t}+v_{x x}-2 u v^{2}=0,
\end{aligned}
$$

in which $u$ and $v$ are treated as independent complex functions of $x$ and $t$.

The dominant balance argument suggests to us the need to introduce the indicial normalization $u=\theta^{-1}$ and to try to find the Laurent $\theta$-series:

$$
\begin{aligned}
& \theta_{x}=a_{0}+a_{1} \theta+a_{2} \theta^{2}+\cdots, \\
& v=\theta^{-1}\left(b_{0}+b_{1} \theta+b_{2} \theta^{2}+\cdots\right),
\end{aligned}
$$

in which $a_{*}, b_{*}, c_{*}$ are functions of $t, \theta_{t}, \theta_{t^{2}}, \ldots$

We substitute (3.14) into (3.13) to get the equation for the leading coefficients

$$
2 a_{0}^{2}-2 b_{0}=0,
$$

and the recursive equation

$$
\begin{aligned}
& (n-4) a_{0} a_{n}+2 b_{n}=F_{n}, \\
& (n-4) a_{0} b_{0} a_{n}+\left[4 b_{0}-\left(n^{2}-3 n+2\right) a_{0}^{2}\right] b_{n}=G_{n},
\end{aligned}
$$

where

$$
\begin{aligned}
& F_{n}=-\delta_{1, n} \mathrm{i} \theta_{t}-\sum_{\substack{j+k=n \\
j, k \neq n}}(j-2) a_{j} a_{k}-\sum_{j+k=n-1}\left(\partial_{x} a_{j}\right)_{k}, \\
& G_{n}=-\mathrm{i}(n-2) \theta_{t} b_{n-1}-2 \sum_{\substack{j+k=n \\
j, k \neq n}} b_{j} b_{k}+2 \sum_{\substack{j+k+l=n \\
j, k, l \neq n}} a_{j} a_{k} b_{l} \\
&-\sum_{\substack{j+k+l=n-1 \\
j \neq n-1}}(j+1) a_{j+1} a_{k} b_{l}-2 \sum_{\substack{j+k+l=n-1 \\
l \neq n-1}}(l+1) a_{j} a_{k} b_{l+1} \\
&+\sum_{j+k+l=n-2}^{\substack{l \neq n-2\\
}}(l+1)(l+2) a_{j} a_{k} b_{l+2}+\sum_{j+k+l=n-2}(k+1)(l+1) a_{j} a_{k+1} b_{l+1} \\
&-\mathrm{i} \partial_{t} b_{n-2}-\sum_{j+k+l=n-1}\left(\partial_{x} a_{j}\right)_{k} b_{l}-2 \sum_{j+k+l=n-1} a_{j}\left(\partial_{x} b_{k}\right)_{l} \\
&+\sum_{\substack{j+k+l=n-1 \\
l \neq 0}} l\left(\partial_{x} a_{j}\right)_{k} b_{l}+2 \sum_{\substack{j+k+l=n-1 \\
j \neq n-1}}(k+l) a_{j}\left(\partial_{x} b_{k}\right)_{l}+\sum_{j+k=n-2}\left(\partial_{x^{2}} a_{j}\right)_{k}
\end{aligned}
$$

and $\left(\partial_{x} a_{j}\right)_{k},\left(\partial_{x} b_{k}\right)_{l},\left(\partial_{x^{2}} a_{j}\right)_{k}$ mean the following: Since $a_{j}$ is a function of $t, \theta_{t}, \theta_{t^{2}}, \ldots$, the partial derivative $\partial_{x} a_{j}$ is a function of $t, \theta_{t}, \theta_{t^{2}}, \ldots$, and the following $\theta$-series

$$
\left(\theta_{x}\right)_{t}=\sum_{j=0}^{\infty}\left[\partial_{t} a_{j}+(j+1) a_{j} \theta_{t}\right] \theta^{j}, \quad\left(\theta_{x}\right)_{t^{2}}, \cdots
$$

Then we find the $\theta$-series for $\partial_{x} a_{j}$, in which $\left(\partial_{x} a_{j}\right)_{k}$ denotes the coefficient of $\theta^{k}$. 
From (3.15), we find $a_{0}=r_{1}(t), b_{0}=r_{1}(t)^{2}$ for an arbitrary nonzero function $r_{1}$ (the first resonance parameter). From (3.16) we further find the $\theta$-series for $u_{x}, v$, and $v_{x}$ :

$$
\begin{aligned}
& u_{x}=-r_{1} \theta^{-2}-\mathrm{i} r_{1}^{-1} \theta_{t} \theta^{-1}-r_{1}^{-3} \theta_{t}^{2}+\frac{\mathrm{i}}{2} r_{1}^{-2} r_{1}^{\prime}+\left(-2 r_{1}^{-1} r_{2}+r_{1}^{-3} \theta_{t^{2}}-\frac{7}{2} r_{1}^{-4} r_{1}^{\prime} \theta_{t}\right. \\
& \left.-3 \mathrm{i} r_{1}^{-5} \theta_{t}^{3}\right) \theta-r_{3} \theta^{2}+\cdots \\
& v=r_{1}^{2} \theta^{-1}+\mathrm{i} \theta_{t}+\left(-\mathrm{i} r_{1}^{-1} r_{1}^{\prime}+r_{1}^{-2} \theta_{t}^{2}\right) \theta+r_{2} \theta^{2}+\left(-\frac{5}{4} r_{1}^{-4} r_{1}^{\prime 2}+\frac{1}{4} r_{1}^{-3} r_{1}^{\prime \prime}-3 \mathrm{i} r_{1}^{-2} r_{2} \theta_{t}\right. \\
& \left.-\frac{19 \mathrm{i}}{2} r_{1}^{-5} r_{1}^{\prime} \theta_{t}^{2}+8 r_{1}^{-6} \theta_{t}^{4}+\mathrm{i} r_{1}^{-4} \theta_{t} \theta_{t^{2}}\right) \theta^{3}+\cdots, \\
& v_{x}=-r_{1}^{3} \theta^{-2}-\mathrm{i} r_{1} \theta_{t} \theta^{-1}+\frac{\mathrm{i}}{2} r_{1}^{\prime}-r_{1}^{-1} \theta_{t}^{2}+\left(\frac{3}{2} r_{1}^{-2} r_{1}^{\prime} \theta_{t}+2 \mathrm{i} r_{1}^{-3} \theta_{t}^{3}\right) \theta+\left(-r_{1}^{2} r_{3}-\frac{21}{4} r_{1}^{-3} r_{1}^{\prime 2}\right. \\
& \left.+\frac{5}{4} r_{1}^{-2} r_{1}^{\prime \prime}-\mathrm{i} r_{1}^{-1} r_{2} \theta_{t}-\frac{53 \mathrm{i}}{2} r_{1}^{-4} r_{1}^{\prime} \theta_{t}^{2}+20 r_{1}^{-5} \theta_{t}^{4}+4 \mathrm{i} r_{1}^{-3} \theta_{t} \theta_{t^{2}}\right) \theta^{2}+\cdots,
\end{aligned}
$$

where $r_{2}$ and $r_{3}$ (the second and the third resonance parameters) are also arbitrary functions of $t$. Then we successively cut $u_{x}$ at $r_{1}, v$ at $r_{2}$, and $v_{x}$ at $r_{3}$ to get the change of variables

$$
\begin{aligned}
& u=\theta^{-1}, \\
& u_{x}=\xi \theta^{-2}, \\
& v=\xi^{2} \theta^{-1}-\mathrm{i} \theta_{t}+\eta \theta^{2}, \\
& v_{x}=\xi^{3} \theta^{-2}-2 \mathrm{i} \xi \theta_{t} \theta^{-1}+\mathrm{i} \xi_{t}+2 \xi \eta \theta+\zeta \theta^{2} .
\end{aligned}
$$

This converts the NLS system (3.13) into the following regular system:

$$
\begin{aligned}
& \theta_{x}=-\xi, \\
& \xi_{x}=2 \theta^{2} \eta-\mathrm{i} \theta_{t}, \\
& \eta_{x}=\zeta, \\
& \zeta_{x}=-2 \theta \eta^{2}-\mathrm{i} \eta_{t} .
\end{aligned}
$$

Note that the mirror system (3.17) is the same as

$$
\begin{aligned}
& \theta_{x x}=-2 \theta^{2} \eta+\mathrm{i} \theta_{t}, \\
& \eta_{x x}=-2 \theta \eta^{2}-\mathrm{i} \eta_{t} .
\end{aligned}
$$

If we change $x$ to $i x$, this becomes the system (3.13) we started with! The transform $(\theta, \eta) \rightarrow(u, v)$ is

$$
\begin{aligned}
& u=\theta^{-1}, \\
& v=\theta_{x}^{2} \theta^{-1}-\mathrm{i} \theta_{t}+\eta \theta^{2} .
\end{aligned}
$$

The inverse transform $(u, v) \rightarrow(\theta, \eta)$ is

$$
\begin{aligned}
& \theta=u^{-1}, \\
& \eta=u_{x}^{2} u^{-1}-\mathrm{i} u_{t}+u^{2} v
\end{aligned}
$$

\section{Convergence of the Laurent series in the Painlevé test}

In [3], we made use of the mirror systems of single high-order completely integrable equations to give conceptual proofs of the convergence of the Laurent series obtained from applying the Painlevé test. The method involves the following steps: 
(1) Convert the Laurent series obtained from the Painlevé test into an initial value problem for the appropriate mirror system.

(2) Apply the Cauchy-Kowalevski theorem to the initial value problem and conclude the convergence of the power series solutions of the mirror system.

(3) The convergent power series solutions of the mirror system lead to convergent Laurent series solutions of the original system, because of the equivalence between the original system and the mirror system.

(4) Compare the Laurent series from step 3 with the series obtained from the Painlevé test explicitly. If the two series are the same up to the order where all the resonances appear, then the two series must be the same (because the whole series are determined by those leading terms).

A consequence of these steps is the convergence of the Laurent series obtained from the Painlevé test. Such convergence can be considered as the justification of the Painlevé test.

The idea works equally well with systems. We present the details only for one case of the Lorenz system. We will also present the outline for the other cases.

\subsection{One case of the Lorenz system}

For the case 1A: $\sigma=\frac{1}{2}, B=1, R=0$, the Painlevé test produces the following formal Laurent series solution:

$$
\begin{aligned}
& X=-2 \mathrm{i} t^{-1}+\frac{\mathrm{i}}{2}+\left(-\frac{\mathrm{i}}{4}+\frac{1}{2} \bar{r}_{1}\right) t+\left(\frac{5 \mathrm{i}}{32}-\frac{3}{8} \bar{r}_{1}\right) t^{2}+\left(-\frac{5 \mathrm{i}}{192}+\frac{1}{16} \bar{r}_{1}+\frac{1}{6} \bar{r}_{2}\right) t^{3}+\cdots, \\
& Y=4 \mathrm{i} t^{-2}-2 \mathrm{i} t^{-1}+\bar{r}_{1}+\left(\frac{3 \mathrm{i}}{8}-\bar{r}_{1}\right) t+\bar{r}_{2} t^{2}+\cdots, \\
& Z=-4 t^{-2}+2 t^{-1}+\mathrm{i} \bar{r}_{1}+\left(-\frac{3}{8}-\mathrm{i} \bar{r}_{1}\right) t+\left(\frac{29}{96}+\frac{5 \mathrm{i}}{8} \bar{r}_{1}+\frac{1}{4} \bar{r}_{1}^{2}-\frac{2 \mathrm{i}}{3} \bar{r}_{2}\right) t^{2}+\cdots,
\end{aligned}
$$

where $\bar{r}_{1}$ and $\bar{r}_{2}$ are the resonance parameters in the Painleve test, and we use $t$ instead of $\left(t-t_{0}\right)$ because of the autonomous system. To show that these series are convergent for small $t$ and arbitrary $\bar{r}_{1}$ and $\bar{r}_{2}$ (the size of $t$ may depend on $\bar{r}_{1}$ and $\bar{r}_{2}$ ), we convert (4.1) to an initial value problem for the mirror system.

We substitute the formal Laurent series (4.1) into the transformation $X=\theta^{-1}$, (3.6) and (3.7) to find the formal power series for $\theta, \xi$ and $\eta$. The computation leads to the following initial data:

$$
\theta(0)=0, \quad \xi(0)=-\frac{5 \mathrm{i}}{4}+3 \bar{r}_{1}, \quad \eta(0)=-\frac{23}{12}-5 \mathrm{i} \bar{r}_{1}+\bar{r}_{1}^{2}+\frac{28 \mathrm{i}}{3} \bar{r}_{2},
$$

for the mirror system (3.8).

By the Cauchy theorem, we know that the initial value problem for the mirror system (3.8) has a power series solution which is convergent in a neighbourhood of zero. We may further find the power series for $\theta, \xi$ and $\eta$ by the usual method such as undetermined coefficients

$$
\begin{aligned}
& \theta=\frac{\mathrm{i}}{2} t+\frac{\mathrm{i}}{8} t^{2}+\left(-\frac{\mathrm{i}}{32}+\frac{1}{8} \bar{r}_{1}\right) t^{3}+\left(\frac{\mathrm{i}}{64}-\frac{1}{32} \bar{r}_{1}\right) t^{4}+\left(\frac{17 \mathrm{i}}{1536}-\frac{5}{128} \bar{r}_{1}\right. \\
& \left.-\frac{\mathrm{i}}{32} \bar{r}_{1}^{2}+\frac{1}{24} \bar{r}_{2}\right) t^{5}+\cdots \\
& \xi=-\frac{5 \mathrm{i}}{4}+3 \bar{r}_{1}+\left(\frac{5 \mathrm{i}}{4}-3 \bar{r}_{1}\right) t+\left(-\frac{31 \mathrm{i}}{96}+\frac{7}{8} \bar{r}_{1}+\frac{\mathrm{i}}{4} \bar{r}_{1}^{2}+\frac{5}{3} \bar{r}_{2}\right) t^{2}+\cdots, \\
& \eta=-\frac{23}{12}-5 \mathrm{i} \bar{r}_{1}+\bar{r}_{1}^{2}+\frac{28 \mathrm{i}}{3} \bar{r}_{2}+\cdots
\end{aligned}
$$


Substituting these series back into $X=\theta^{-1}$, (3.6) and (3.7), we find the convergent (i.e., not just formal) power series for $X, Y$ and $Z$. The computation shows that the result is exactly (4.1). Thus we conclude that the Laurent series (4.1) are convergent.

\subsection{Other cases and systems}

For the case 1B ( $\left.\sigma=1, B=2, R=\frac{1}{9}\right)$ of the Lorenz system, the Painlevé test produces the following formal Laurent series solution:

$$
\begin{aligned}
& X=-2 \mathrm{i} t^{-1}+\frac{2 \mathrm{i}}{3}+\left(-\frac{2 \mathrm{i}}{3}+\bar{r}_{1}\right) t+\left(\frac{32 \mathrm{i}}{27}-2 \bar{r}_{1}\right) t^{2}+\left(-\frac{32 \mathrm{i}}{81}+\frac{2}{3} \bar{r}_{1}+\frac{1}{3} \bar{r}_{2}\right) t^{3}+\cdots \\
& Y=2 \mathrm{i} t^{-2}-2 \mathrm{i} t^{-1}+\bar{r}_{1}+\left(\frac{46 \mathrm{i}}{27}-3 \bar{r}_{1}\right) t+\bar{r}_{2} t^{2}+\cdots \\
& Z=-2 t^{-2}+\frac{4}{3} t^{-1}+\frac{2}{9}+\mathrm{i} \bar{r}_{1}+\left(-\frac{20}{27}-\frac{4 \mathrm{i}}{3} \bar{r}_{1}\right) t+\left(\frac{142}{81}+\frac{8 \mathrm{i}}{3} \bar{r}_{1}+\frac{1}{2} \bar{r}_{1}^{2}-\frac{2 \mathrm{i}}{3} \bar{r}_{2}\right) t^{2}+\cdots
\end{aligned}
$$

The convergence of the series may be proved by considering the following initial value:

$$
\theta(0)=0, \quad \xi(0)=-\frac{16 i}{9}+3 \bar{r}_{1}, \quad \eta(0)=-\frac{1336}{81}-\frac{80 \mathrm{i}}{3} \bar{r}_{1}-2 \bar{r}_{1}^{2}+\frac{20 \mathrm{i}}{3} \bar{r}_{2},
$$

for the corresponding mirror system.

For the case $2\left(\sigma=\frac{1}{3}, B=0, R\right.$ arbitrary), we should consider the following initial data:

$$
\theta(0)=0, \quad \xi(0)=3 \bar{r}_{1}, \quad \eta(0)=-\frac{2}{3} \bar{r}_{1}^{2}+\frac{20 \mathrm{i}}{3} \bar{r}_{2},
$$

for the corresponding mirror system. This leads to the convergence of the following Laurent series obtained from the Painlevé test:

$$
\begin{aligned}
& X=-2 \mathrm{i} t^{-1}+\frac{1}{3} \bar{r}_{1} t+\frac{2}{9} \bar{r}_{2} t^{2}+\cdots \\
& Y=6 \mathrm{i} t^{-2}-2 \mathrm{i} t^{-1}+\bar{r}_{1}+\frac{1}{3} \bar{r}_{1} t+\bar{r}_{2} t^{2}+\cdots \\
& Z=-6 t^{-2}+4 t^{-1}-1+R+\mathrm{i} \bar{r}_{1}-\frac{4 \mathrm{i}}{3} \bar{r}_{1} t+\left(\frac{1}{6} \bar{r}_{1}^{2}-\frac{2 \mathrm{i}}{3} \bar{r}_{2}\right) t^{2}+\cdots
\end{aligned}
$$

For the branch of the Rikitake model considered in section 3.2 in the case $\alpha=\beta=0$, we solve the mirror system (3.12) with the initial data

$$
\theta(0)=0, \quad \xi(0)=2 \mathrm{i} \bar{r}_{1}+\bar{r}_{2}, \quad \eta(0)=-\bar{r}_{1}+2 \mathrm{i} \bar{r}_{2} .
$$

This leads to the convergence of the following Laurent series:

$$
\begin{aligned}
& X=\mathrm{i} t^{-1}+\left(-\bar{r}_{1}+\mathrm{i} \bar{r}_{2}\right) t+\cdots, \\
& Y=\mathrm{i} t^{-1}+\bar{r}_{1} t+\cdots, \\
& Z=-t^{-1}+\gamma+\bar{r}_{2} t+\cdots,
\end{aligned}
$$

obtained in the Painlevé test for the Rikitake model. The convergence of the Laurent series in other cases of the Rikitake model can be proved similarly.

By solving the mirror system (3.17) for the NLS system (3.13) with the following initial data along the initial manifold $x=\psi(t)$ :

$$
\begin{aligned}
& \theta=0 \\
& \xi=\bar{r}_{1}^{-1} \\
& \eta=\frac{1}{2} \psi^{\prime} \bar{r}_{1}^{\prime}+\frac{1}{4} \psi^{\prime \prime} \bar{r}_{1}^{\prime}+6 \bar{r}_{2} \\
& \zeta=\frac{1}{72} \psi^{\prime 4} \bar{r}_{1}+\frac{\mathrm{i}}{12} \psi^{\prime} \psi^{\prime \prime} \bar{r}_{1}+\frac{1}{9} \bar{r}_{1}^{-1} \bar{r}_{1}^{\prime 2}-\frac{\mathrm{i}}{18} \psi^{\prime 2} \bar{r}_{1}^{\prime}+\frac{1}{12} \bar{r}_{1}^{\prime \prime}-2 \mathrm{i} \psi^{\prime} \bar{r}_{2}+10 \bar{r}_{3},
\end{aligned}
$$


where $\psi, \bar{r}_{1}, \bar{r}_{2}$, and $\bar{r}_{3}$ are arbitrary analytic functions and $\bar{r}_{1} \neq 0$, we can prove the convergence of the Laurent series

$$
\begin{aligned}
& u=\bar{r}_{1}(x-\psi)^{-1}+\frac{\mathrm{i}}{2} \psi^{\prime} \bar{r}_{1}+\left(-\frac{1}{12} \psi^{\prime 2} \bar{r}_{1}+\frac{\mathrm{i}}{6} \bar{r}_{1}^{\prime}\right)(x-\psi)+\bar{r}_{2}(x-\psi)^{2}+\bar{r}_{3}(x-\psi)^{3}+\cdots \\
& v=\bar{r}_{1}^{-1}(x-\psi)^{-1}-\frac{\mathrm{i}}{2} \psi^{\prime} \bar{r}_{1}^{-1}+\left(-\frac{1}{12} \psi^{\prime 2} \bar{r}_{1}^{-1}+\frac{\mathrm{i}}{6} \bar{r}_{1}^{-2} \bar{r}_{1}^{\prime}\right)(x-\psi) \\
&+\left(-\frac{1}{4} \psi^{\prime \prime} \bar{r}_{1}^{-1}-\bar{r}_{1}^{-2} \bar{r}_{2}\right)(x-\psi)^{2} \\
&+\left(\frac{\mathrm{i}}{6} \psi^{\prime} \psi^{\prime \prime} \bar{r}_{1}^{-1}+\frac{1}{12} \bar{r}_{1}^{-3} \bar{r}_{1}^{\prime 2}-\frac{1}{12} \bar{r}_{1}^{-2} \bar{r}_{1}^{\prime \prime}+\bar{r}_{1}^{-2} \bar{r}_{3}\right)(x-\psi)^{3}+\cdots
\end{aligned}
$$

These are the Laurent series obtained in the Painlevé test.

\section{Acknowledgments}

We would like to thank the referees for many suggestions that improved the presentation of the paper.

\section{References}

[1] Ablowitz M J, Ramani A and Segur H 1980 A connection between nonlinear evolution equations and ordinary differential equations of P-type I: II J. Math. Phys. 21 715-21

Ablowitz M J, Ramani A and Segur H 1980 J. Math. Phys. 21 1006-15

[2] Bountis T, Ramani A, Grammaticos B and Dorizzi B 1984 On the complete and partial integrability of nonHamiltonian systems Physica A 128 268-88

[3] Hu J and Yan M 1999 The mirror systems of integrable equations Stud. Appl. Math. to appear

[4] Hu J and Yan M 1999 Analytical aspects of the Painlevé test submitted

[5] Painlevé P 1900 Mémoires sur les équations différentielles dont l'intégrale générale est uniforme Bull. Soc. Math. France 28 201-61

[6] Ramani A, Grammaticos B and Bountis T 1989 The Painlevé property and singularity analysis of integrable and nonintegrable systems Phys. Rep. 180 159-245

[7] Segur H 1980 Soliton and inverse scattering transform Lectures given at the International School of Physics (Enrico Fermi, Varenna, Italy, July 7-19)

[8] Weiss J, Tabor M and Carnevale G 1983 The Painlevé property for partial differential equations J. Math. Phys. 24 522-6 